\title{
Increasing Virulence of Vulvovaginal Candididiasis (VVC) in the Modern Era with Reducing Immunity by Different Candida Species Along with Increasing Resistance and Management -An Update
}

\author{
Kulvinder Kochar Kaur ${ }^{1 *}$, Gautam Allahbadia ${ }^{2}$ and Mandeep Singh ${ }^{3}$ \\ ${ }^{1}$ Scientific Director, Centre for Human Reproduction, Nagar, Jalandhar, Punjab, India \\ ${ }^{2}$ Scientific Director, Ex-Rotunda-A Centre for Human reproduction, Kalpak Garden, Bandra, Mumbai, India \\ ${ }^{3}$ Consultant Neurologist, Swami Satyanand Hospital, Near Nawi Kachehri, Baradri, Jalandhar, Punjab, India \\ *Corresponding Author: Kulvinder Kochar Kaur, Scientific Director, Centre For Human Reproduction, Nagar, Jalandhar, Punjab, India.
}

Received: October 03, 2019; Published: November 04, 2019

DOI: 10.31080/ASMI.2019.02.0421

\begin{abstract}
Although Candida yeasts are present as vaginal microbiota, presence of them always doesn't lead to clinical symptoms. Interactions between host defense functions besides synergism with a variety of microorganisms from the vaginal mucosa are the ones which maintain balance of vaginal surroundings. On breakdown of the vaginal ecosystem, overgrowth of Candida yeasts is helped that results in development of vulvovaginal candidiasis (VVC). VVC is an opportunistic mucosal infection being $2^{\text {nd }}$ commonest vaginal infection that affects women in reproductive age, affecting $75 \%$ of these women of which $50 \%$ at least get recurrence of the same infection once. Recently globally it has been found candidiasis is becoming a threat especially for women with poor immunity specially those with HIV infection. Further not only the original conventional candida albicans is becoming responsible for the infection with candida but other species like C. tropicalis and C. parapilasis and C. gabratus ar becoming the pathogenic organisms involved in causing VVC not only in nonpregnant women but also in women with pregnancy where it is becoming a threat for the fetus as well. Here we review the role of various Lactobacillus species, their biosurfactant properties and role of moonlighting proteins in the virulence of candida, besides further measures to reduce the virulence and treatment of VVC effectively.
\end{abstract}

Keywords: VVC; Candida Albicans; C. Tropicalis and C. parapilasis; Lactobacillus Species; Probiotics; Ketoconazole; Fluconazole; HIV

\section{Introduction}

Although Candida yeasts are present as vaginal microbiota, presence of them always doesn't lead to clinical symptoms. Interactions between host defense functions besides synergism with a variety of microorganisms from the vaginal mucosa are the ones which maintain balance of vaginal surroundings [1]. On breakdown of the vaginal ecosystem, overgrowth of Candida yeasts is helped that results in development of vulvovaginal candidiasis (VVC) [2]. VVC an opportunistic mucosal infection being 2nd commonest vaginal infection that affects women in reproductive age [3], affecting $75 \%$ of women once in a lifetime, and $50 \%$ of them suffering a minimum of a single recurrence $[3,4]$. Initial symptoms of VVC are pruritus and soreness of the vulva, dysuria, white cottage cheese like vaginal discharge along with dyspareunia. Quality of life gets affected by VVC besides predisposing to development of HIV susceptibility [5]. Though very little mortality is there with VVC symptoms =>marked morbidity mainly in women infected with human immunodeficiency virus (HIV) [6]. Moreover, women having vaginal colonization of Candida spp during 2 nd trimester of pregnancy have less neonatal birth weights and > preterm birth in contrast to colonization by other microbes [7].

\section{Methods}

Thus we used the search engine PubMed using the Me SH terms like vulvovaginal candidiasis; candida species; lactobacillus species; drugs for treatment of VVC; Drug resistance; virulence; VVC in pregnancy; moonlighting proteins; probiotics; immunodeficiency and VVC.

\section{Results and Discussion}

We found a total of 3846 articles out of which we selected 50 articles for this review. No meta-analysis was done.

\section{Role of Lactobacillus species in VVC}

Frequent coitus, persons' susceptibility, antibiotic use, along with contraceptive and spermicidal utilization, pregnancy, DM, and immunosuppression predispose to V V C development [5]. Usually the cause of VVC is candida albicans, but there has been marked 
Increasing Virulence of Vulvovaginal Candididiasis (VVC) in the Modern Era with Reducing Immunity by Different Candida Species Along with Increasing Resistance and Management -An Update

rise in V VC being caused by other candida spp [6,7] These include Candida glabrate, Candida parasitosis, Candida krusei, Candida tropicalis, that have been identified with greater frequency [8-0]. With > infections with other Candida spp the rate of recurrence along with resistance has started occurring $[10,11]$.

Normally Lactobacillus species colonize the human body, thus become a part of the resident microbiota, not causing any harm to host. During healthy state vaginal microbiota, Lactobacillus species are partly the most abundant of the micro-organisms [12]. Lactobacillus species regulate the increased proliferation of a lot of pathogens that have the capability of producing organic acids along with antimicrobial compounds like hydrogen peroxide, bacteriocins, and surface active compounds, that include biosurfactants (BSs) either by auto aggregation or by competing with each other for nutrients along with adhering areas in the vaginal epithelium [13]. Still there is debate about the a etiopathogenesis of $V$ V C. Increased susceptibility related to genetics, pregnancy, antibiotic use, along with contraceptive and spermicidal usage, Frequent coitus, immunosuppression are factors representing the increased likelihood of V V C development [2,14]

Variety of surface active substances can be synthesized by BSc's that have low molecular weight s. Surfactant along with emulsifying properties are expressed by BSc's and thus possess the capacity to reduce the interface between2 phases of a heterogenous system, further they possess antibacterial, antifungal, anti adhesive, and antibiofilm properties, besides having the ability to be utilized as major immunomodulatory molecules or in vaccines and gene therapy [15].

By definition probiotics are "live organisms", which once given in enough quantities, give a health benefit for the host [16]. Various researchers have documented the utilization of BSc's that are synthesized by lactic acid bacteria (LAB) in both food along with health industries [17] In the food industries it can be utilized as a treatment for food contact surfaces, hence preventing biofilm development, food additive /ingredient, and in residues therapy [18]. In health industry, their use might be associated with anti adhesive function, that prevent the adhesion of pathogenic organisms to solid surfaces, like silicone rubber, surgical implants along with vinyl urethral catheters, or biological surfaces, like (urogenital and intestinal tract epithelial cells ) $[17,19,20]$. Also, BS may get utilized in pharmaceutical fields in the form of agents for respiratory failure, immunological adjuvants. Other than that, BS get utilized for respiratory failure as pharmacological agents, an immunological adjuvants, recovery of intracellular products, antimicrobial activity, antiviral activity, anticancer effect, besides as substances to stimulate fibroblast metabolism [21]. LAB interference In pathogen colonization in colonization of pathogen takes place through multiple modes of action that includes production of BS [22].

An other mechanism for interference with or preventing colonization through pathogen microorganisms is use of surface active compounds, and thus avoid the progression of infections. Currently Lactobacillus species have gained importance in view of antagonistic actions against multiple pathogens that they possess, which points to potential therapeutic or prophylactic use for some infectious diseases [23]. Carmo., et al. had earlier shown that Lactobacillus fermentum ATCC23271 showed antagonistic action on candida spp in vitro and further inhibited yeast adhering to HeLa cells and mucin [24]. But the actions and anti- candida mechanisms of Lactobacillus BS's are still not clear, particularly those related to resident Lactobacillus spp.

Thus dos Santos., et al. examined the antagonistic action of vaginal Lactobacillus spp on candida albicans to confirm if active substances of Lactobacillus spp possessed antifungal and ant virulence activity. On antagonism assay it was found that 15/20 Lactobacillus strains possessed an inhibitory action against candida albicans. Biosurfactants showed surface - tension-decreasing property and best results were obtained for Lactobacillus gasseri 1 and Lactobacillus rhamnosus ATCC 9595, Lactobacillus Acidophillus ACTT 4356, and Lactobacillus paracasei 11, that produced Biosurfactants which reduced candida albicans adhesion and prevented biofilm. Most optimum results were achieved in the pre-incubation assay for L. gasseri 1 and L. paracasei 11. In total, Lactobacillus strains displayed significant anti- candida action, and their Biosurfactants demonstrated significant anti- adhesion and anti- biofilm effects against candida albicans. For demonstrating biosurfactant (BS) safety needs to be evaluated through cytotoxic assays [25].

Istanbullu., et al. evaluated the antifungal actions of different lactic acid bacteria which were identified from the vagina of healthy women on some Candida spp isolated from the vagina were examined by agar diffusion technique. Most of the lactic acid bacteria which belongto the species of Lactobacillus crisbatus. L. fermentum, L. Acidophillus, L. paracesei subsp. Paracesei L. pentosus and L. plantarum demonstrated antifungal actions in differing ratios against $C$. albicans, C. glabrata, and C. tropicalis strains found from the vagina Thus concluding that the lactic acid bacteria are useful microorganisms that are related to various probiotic characteristics. With this in mind their lactic acid bacteria isolates with great antifungal activity might be important candidates as probiotic microorganisms in inhibiting the vaginal candidiasis, that is one of the most prevalent difficulties, or in the protection against candidiasis. They plan to continue studies on this area [26]. 


\section{Efficacy of various antifungal agents}

Various virulence determinants and increasing resistance against antifungal therapy have led to the pathogenicity of candida albicans. But association in between resistance profiles along with patterns of virulence of candida albicans has not been examined properly. Thus El-Houssaini., et al. collected vaginal swab specimens from 65 subjects, and studied the species level. They checked the susceptibility of isolates against different antifungal drugs like amphotericin B, nystatin, clotrimazole, fluconazole, voriconazole and micafungin), along with their minimum inhibitory concentrations (MIC's) as per the Clinical and Laboratory Standards Institutes (CLSI) guidelines Regarding virulence patterns studied were secreted hydrolases (phospholipase, aspartyl protease, and haemolysin), cell surface hydrophobicity, and biofilm production. They correlated resistance profiles and virulence patterns of the candida albicans isolates examined. Different levels of resistance and virulence patterns was displayed by the isolates of candida albicans examined. Moreover, a significant $(\mathrm{p}<0.05)$ positive correlation between amphotericin B MICs and biofilm production was found. But significant $(\mathrm{p}<0.05)$ negative association was found between fluconazole and voriconazole MICs and cell surface hydrophobicity along with biofilm production. Furthermore significant $(\mathrm{p}<0.05)$ negative association was observed between voriconazole MICs and aspartyl protease synthesis. Thus concluding how this research showed significant association between resistance profiles and virulence patterns of candida albicans isolates that were obtained from vulvo vaginal specimens [27].

\section{VVC in pregnancy}

Various publications have demonstrated that candida prevalence is > in pregnant women as compared to non pregnant women and has a tendency to rise as pregnancy proceeds [28]. Data coming out further shows that VVS during pregnancy may be related to > risk of premature rupture of membranes (PROM), preterm labor, chorioamnionitis and congenital cutaneous candidiasis [29].

Causes of > risk of VVC in pregnancy are associated with physiologic alterations like a reduced cellular immunity, increased hormone levels, decreased vaginal $\mathrm{pH}$, and elevated vaginal glycogen concentrations [28, 30]. Further colonization of candida is also thought to be secondary to use of broad spectrum antibiotic and has been recurrently correlated with a reduced colonization of Lactobacillus, possibly secondary to interference with epithelial binding sites [5]. Once the balance between candida, normal bacterial flora along with immune defense mechanism goes haywire, and thus colonization gets replaced by infection. As per most reports candida albicans causes largest numbers of VVC $=>85-90 \%$ of episodes where vaginal candidiasis presents with symptoms [5].
Besides candida albicans, other candida species that are now being shown as the causative agents for VVC, are C. glabrata, C. tropicalis and C. krausei, but have lot of differences as far as epidemiology, virulence, and antifungal drug susceptibility is concerned $[3,4,31]$.

In neonates candidiasis that has invading properties has started causing late onset sepsis, with mortality rates approaching as much as $25-35 \%$ [32]. There has been an increase by 11 times in the past 15 years, with candida species being responsible as the $3^{\text {rd }}$ most common organism that is isolated in low birth weight infants having late onset sepsis [32].

Thus Ghaddar., et al. tries to estimate the prevalence of risk factors of VVC in pregnant women at 35 - 37 weeks of pregnancy. They also tried to find any probable association between VVC and vaginal colonization with separate organisms like Group B Streptococci (GBS) and bacterial vaginosis. Thus across 1 year period they collected high vaginal swabs from pregnant women presenting during their routine antenatal check (ANC) up in separate polyclinics in Beirut and South Lebanon. Swabs got cultured on Sabourad Dextrose Agar, and identified Candida isolates with the use of Chromatis Candida medium and Germ tube test. They identified V V C in 44. $8 \%$ of samples with C. glabrata (44. 4\%) and candida albicans (43. $4 \%$ ) being the maximum isolated species. Roughly $50 \%$ of pregnant women (57.7\%) had coinfection of candida and bacterial vaginosis, in comparison to $26 \%$ of them having candida species with GBs. No association was observed between the presence of VVC and demographic clinical, medical and reproductive health properties of pregnant women. Reversely those who participated with history of previous miscarriages and those that had been hospitalized in the last $12 \mathrm{mths}$ had $>$ chances of acquiring vaginal, $C$. krausei infection as compared with other Candida $\operatorname{spp}(\mathrm{p}=0.0316$ and $\mathrm{p}=0.0042$, respectively). Thus conclusions drawn were that the prevalence of VVC in pregnant women has taken a rising trend in their community. Hence routine medical examination with regular screening for candidiasis in the antenatal care programmes is recommended for treating the disease and avoiding its complications [33].

\section{Role of moon lighting proteins}

Pathogenic microorganisms have the ability to adjust easily to variety of environmental surroundings, that they exploit when colonizing new infectious niches in a host organism. At the time of the start and progression of infectious process, these microbes especially have to deal with variations in temperature, $\mathrm{pH}$, and osmolality, different oxygen and nutrients, oxidative stress along with the host immune response. Besides using full suite of typical virulence factors, like hydrolytic enzymes, toxins or adhesins, less apparent or not well known actions which allow survival of the mi- 
crobes in the presence of unsuitable environmental surroundings. Cell surface enzymes which have their origin in the cytoplasm in the pathogens is an unusual mode, involved basically in necessary intracellular metabolic processes like the citric acid cycle, or the pentose phosphate pathway [34]. A cellular position, which is completely separate from the initial points to some other function of these factors which is not the result of gene fusion or splicing variations. The phrase "moonlighting proteins" was thus given because of this [35]. What the functional point involved here is the use in a successful manner by different species of pathogenic bacteria, where surface exposed 'moonlighting proteins take an important part in the adhesion to host cells and tissues, with binding of different proteinaceous targets within the host organism, evading the immune system [36].

Same process has also been revealed for yeast like fungi from Candida genus. Thus these microorganisms have the ability to become a threat to humans which can not only result in repeated superficial infections of the skin along with mucosal surfaces, but can also become invasive, causing systemic infections mainly if the host has impaired immunity [37]. Various moonlighting proteins that are present on cell surface of $C$. albicans, C. tropicalis and $C$. parapilasis have been recognized as binding partners for human extracellular matrix proteins [38], plasminogen and complement system and kinin generating system $[39,40]$, and also as molecules which mediate the fungal cells binding to human cells [41]. Furthermore, moonlighting proteins from a variety of candida spp can act in bringing about the adhesion of fungi to medical devices that are made up of silicone or polyvinyl chloride [42].

A probable protective role of surface exposed moonlighting proteins has been hypothesized, that as a response to oxidative stress caused by $\mathrm{H}_{2} \mathrm{O}_{2}$ in $C$. albicans $C$. krausei and $C$. parapilasis. Different metabolic enzymes that are present in the cell wall of these fungi grown in liquid media or in sessile cells that formed biofilms might be depicting a primary line of defense against ROS produced at the time of phagocytic respiratory burst along with the typical antioxidant systems was shown [43]. This action in conjunction with the capacity to adhere to various biotic and abiotic surfaces and for affecting the action of plasma proteolytic cascades, that can markedly add to the virulence of candida organisms. On host invasion along with colonization of new niches by fungal cells needs a dynamic adjustment of these microorganisms to the new environmental surroundings, that includes variation in their cell wall proteome [44]. But considering the marked alteration in frequency of infection initiated by a specific candida spp. Have been seen and the species besides $C$. albicans, in particular C. glabrata, C. parapilasis and C. krausei have been found to be the cause of superficial and invasive candidiasis [45], special emphasis needs to be made on the pathogenicity associated contribution of these species, since these are being revealed in a > amount as an important threat for those subjects having low immunity. Regarding C. albicans the cell wall structure and changes in its proteome is well studied in a relative term, but few publications on the composition of the cell walls of other species of candida genus exist. Of the proieins that are there in the cell surface of $C$. albicans cells, many moon lighting proteins have been consecutively reported, that include, enolase (Eno 1), glyceraldehydes 3 -phosphate dehyrogenase (Tdh3), alcohol dehyrogenase (Adh1), phosphoglycerate kinase (Pgk1), transaldolase (Tal1), pyruvate decarboxylase (Pdc11) and others [46]. In view of marked differences in virulence activities between species can be present, that includes separate protein exposition at the cell surface, this may cause differences in pathogen-host interaction, a rise in the incidence of candidiasis between species other than the well studied $C$. albicans and higher problems in the therapy of such infections. Thus Karkowsa-Kuleta., et al. used cell surfaces having with the help of trypsin and a shotgun proteomic approach to describe the surface exposed proteins of 3 important nonalbicans candida spp. - C. glabrata, C. parapilasis and $C$. tropicalis. These proteinaceous parts were isolated following the growth of the fungal cells in different culture medium that included artificial urine and vaginal -mimicking medium, under aerobic conditions and in anaerobically rich YPD medium. Various known proteins taking part in cell wall maintenance and fungal pathogenesis were revealed at the cell surface just like a number of atypical cell wall parts - enolase (Eno 1), pyruvate decarboxylase (Pdc11), glyceraldehydes 3 - phosphate dehyrogenase (Tdh3), that are the so called moonlighting proteins. Importantly many of these proteins displayed marked upregulation at the cell surface in growth media simulating the conditions of infection as compared to defined synthetic medium. Thus concluding that moonlighting proteins are expressed under different conditions at the cell walls of the C. glabrata, C. parapilasis and C. tropicalis fungal pathogens. This points that a probable universal surface related part of these factors is present in the physiology of these fungi and the pathology of the infection they lead to [47].

\section{Role of novel agents}

Ibrexafungerip (IBX) (earlier SCY - 078), represents a novel glucan synthase inhibitor whose oral availability is being tested for efficacy against VVC. Bioavailability along with in vitro activity represent important efficacy indicators, but the susceptibility assays don't predict correctly in the acidic surroundings like in the vagina. Larkin., et al. studies done were 3 fold i) pharmacokinetic study after oral intake in a murine model ii) susceptibility testing of isolates 
Increasing Virulence of Vulvovaginal Candididiasis (VVC) in the Modern Era with Reducing Immunity by Different Candida Species Along with Increasing Resistance and Management -An Update

from a phase 2 VVC clinical trial by CLSIM 27-A4 technique; and iii) susceptibility testing of candida albicans and C. glabrata removed from this trial group in RPMI 1640 adjusted to 3 separate $\mathrm{pH}$ values, 7.0, 5.72, and 4.5, in comparison to susceptibility testing for candida and fluconazole. IBX easily accumulated in vaginal tissues and secretions after oral intake. High in vitro action was shown against candida strains received at baseline and end of study visits. Furthermore the geometric mean (GM) values for IBX at $\mathrm{pH} 4.5$ were markedly lower as compared to $\mathrm{pH} 7.0$ and 5.72. The $\mathrm{MIC}_{92}$ values of candida continues to be same, irrespective of $\mathrm{pH}$ values, while those of fluconazole increased with lesser $\mathrm{pH}$ values. IBX can reach target tissues after orally giving at pharmacologically level that are meaningful. High in vitro action was shown by IBX, without resistance, after consecutive exposure in the time of the clinical trial. Significantly action of IBX in an acidic medium points to a therapy advantage of this novel antifungal in the therapy of vaginal candida infections [48].

\section{Role of ketoconazole (KTZ) with special delivery system}

Ketoconazole (KTZ) represents an antifungal drug having poor water solubility along with penetration capacity, is usually not effective in deep seated candidiasis. In view of these problems, Abd Ellah., et al. tried to develop a novel multifunctional carrier for KTZ through encapsulation for KTZ/ $\beta$ - Cyclodextrin ( $\beta$-CD) co-ground mixture into chitosan/gellan gum gel-flakes (thread like and polygonal structures). Evaluation studies showed the presence of electrostatic -derived complexes between negatively charged gellan gum and positively charged chitosan. Gel flakes were put in insitu gel of pluronic (F-127). On the basis of gelation temperature (Tgel), viscosity and release studies; further they examined selected formulations, demonstrating significant in vitro anti-candida action. Inspite of decreased dosage regimen $(50 \mathrm{mg}$ /day for 3 days, KTZ flakes in situ gel was equally effective as Gynoconazol vaginal cream ${ }^{\circledR}(80, \mathrm{mg}$ and virulence terconazole /day for 3 days) with regard to relief of complaints along with removal of candida. KTZ carrier possessing umpteen functions was on the basis of good spreading because of which It coated vagina evenly in view of fleeflowing characteristics at the time of applying it, flakes getting entangled in the folds of vaginal epithelium, release was sustained along with > penetration capacity of KTZ for reaching deep - seated infections. Thus concluding, flakes in situ gel might be thought of as a very good method for treating vaginal Candidiasis [49].

In a murine model of vulvo vaginal candidiasis, induced by candida albicans Esposito., et al. showed that the topical treatment of grape seed dry extract and pea in a non pharmacological device significantly decreased the fungal burden in vagina and preserved Vaginal tissue architecture from candida albicans infection. Thus concluding the probable benefit of the association between grape and pea extract that is present in the device. In all these results pointed to the device being helpful antifungal agent and helpful synergistic device along with fluconazole in the treatment of clinical vulvo vaginal candidiasis by candida albicans biofilms [50].

\section{Conclusion}

Thus here we have emphasized on how besides candida albicans other candida species like C. glabrata, C. parapilasis and $C$. tropicalis are becoming increasingly pathogenic in the causation of VVC not only in nonpregnant women but also in pregnant women where their presence is associated with PROM, preterm labour along with low birth weight and greater cause of deep seated candidiasis in neonates and sepsis in the newborn. In women having lower immunity candida spp has become more dangerous and virulent besides resulting in greater resistance to the conventional antifungal agents like clotrimazole, fluconazole. Role of Lactobacillus species present in parabiotics is one suggested method of treating these infections besides role of biosurfactants produced by them highlighted. Further role of moonlighting proteins is highlighted being present on the cell surface increasing adhesiveness etc. Different delivery systems have been advocated for making drugs like ketoconazole and others become effective like in gel flake form or device that ensures smooth coating of the vagina besides the drug getting trapped in the folded vaginal epithelium.

\section{Bibliography}

1. Larsen B and Monif GRG. "Understanding the bacterial flora of the female genital tract”. Clinical Infectious Diseases 32 (2001): e69-e77.

2. Parolin C., et al. "Isolation of vaginal Lactobacilli and characterization of anti- Candida Activity". PLoS ONE 10 (2015): e0131220.

3. Aguin TJ and Sobel JD. "Vulvovaginal candidiasis in pregnancy". Current Infectious Disease Reports 17.6 (2015): 462.

4. Alfouzan W., et al. "Species, spectrum and antifungal susceptibility profile of vaginal isolates of Candida in Kuwait". Journal de Mycologie Medicale 25.1 (2015): 23-28.

5. $\quad$ Sobel JD. “Vulvovaginal candidiasis". Lancet 369.9577 (2007): 1961-1971.

6. Xie HY., et al. "Probiotics for Vulvovaginal candidiasis in non pregnant women". Cochrane Library: Cochrane Reviews (2017): 11CD010496. 
Increasing Virulence of Vulvovaginal Candididiasis (VVC) in the Modern Era with Reducing Immunity by Different Candida Species Along with Increasing Resistance and Management -An Update

7. Holzer I., et al. "The colonization with Candida Species is more harmful in the second trimester of pregnancy". Archives of Gynecology and Obstetrics 295 (2017): 891-895.

8. $\quad$ Alizadeh M., et al. "Identification of Candida Species isolated from Vulvovaginitis using matrix assisted laser desorption ionization time of flight mass spectrometry". Current Medical Mycology 3 (2017): 21-25.

9. Nejat ZA., et al. "Identification and Antifungal Susceptibility Patterns of Non albicans Candida Species Isolated from Vulvovaginal candidiasis". Iranian Biomedical Journal 22 (2018): 33-41.

10. Goncalves B., et al. "Vulvovaginal candidiasis:epidemiology,m icrobiology and risk factors". Critical Reviews in Microbiology 42 (2016): 905-927.

11. Sherry L., et al. "Biofilms formed by isolates from recurrent Vulvovaginal candidiasis patients are heterogenous and insensitive to fluconazole". Antimicrobial Agents and Chemotherapy 61 (2017): e01065-e01117.

12. Ravel J., et al. "Vaginal microbiome of reproductive age women". Proceedings of the National Academy of Sciences of the United States of America 108 (2011): 4680-4687.

13. Borges S., et al. "The role of Lactobacilli and probiotics in maintaining vaginal health". Archives of Gynecology and Obstetrics 289 (2017): 479-489.

14. Babula 0., et al. "Frequency of interleukin 4 (IL-4)589 Gene polymorphisms and vaginal concentrations of IL-4, Nitric oxide,and mannose Binding Lectin in Women with Recurrent Vulvovaginal candidiasis". Clinical Infectious Diseases 40 (2005): 1258-1262.

15. Rodriques LR., et al. "Physicochemical and functional characterization of biosurfactant produced by Lactobacillus lactis 53". Colloids and Surfaces B: Biointerfaces 49 (2006): 79-86.

16. Hill C., et al. "Expert Consensus document.The International Scientific Association for Probiotics and Prebiotics Consensus statement on the scope and appropriate use of the term probiotic". Nature Reviews Gastroenterology and Hepatology 11 (2014): 506-514.

17. Sharma D., et al. "Isolation and functional characterization of novel biosurfactant produced by Enterococcus faecium". Springer Plus 4 (2015): 1.
18. Nitschke M and Silva SSE. "Recent food applications of microbial surfactants". Critical Reviews in Food Science and Nutrition 58 (2017): 631-638.

19. Reid G., et al. "Urogenital lactobacilli Probiotics, reliability and regulatory stress". Journal Dairy Stress 84 (2001): E164E169.

20. Saharan BS., et al. "A review on biosurfactants: fermentation,current developments and perspectives". Journal of Genetic Engineering and Biotechnology (2011):1-14.

21. Shekhar S., et al. "Biosurfactants producing microbes and their Potential Applications: A Review". Critical Reviews in Environmental Science and Technology 45 (2014): 1522-1554.

22. Santos DK., et al. "Synthesis and evaluation of biosurfactant produced by Candida lipolytica using animal fat and corn sheep liquor". Journal of Petroleum Science and Engineering 105 (2013): 43-50.

23. Wang S., et al. "Antimicrobial compounds produced by vaginal Lactobacillus crispatus are able to strongly inhibit candida albicans growth, hyphal formation and regulate virulencerelated Gene Expression". Frontiers in Microbiology 8 (2017): 564.

24. Carmo MS., et al. "Lactobacillus fermentum ATCC23271 displays in vitro inhibitory activities against Candida spp". Frontiers in Microbiology 7 (2016): 1722.

25. Dos-Santos CI., et al. "Antifungal and Ant virulence Activity of Vaginal Lactobacillus Spp. Products against Candida Vaginal Isolates". Pathogens 8 (2019): 150.

26. Istanbullu Er S., et al. "Anti- Candidal activities of lactic acid bacteria isolated from the vagina". Turkish Journal of Medical Sciences 49.1 (2019): 375-383.

27. El-Houssaini HH., et al. "Correlation between Antifungal resistance and virulence factors in candida albicans recovered from Vaginal specimens". Microbial Pathogenesis 128 (2019): 13-19.

28. Guzel AB., et al. "An evaluation of risk factors in pregnant women with Candida Vaginitis and the diagnostic value of simultaneous vaginal and rectal sampling". Mycopathologia 172.1 (2011): 25-36.

29. Meisozo T., et al. "Intrauterine candidiasis. Report of 4 cases". Archives of Gynecology and Obstetrics 278.2 (2008): 173-176. 
Increasing Virulence of Vulvovaginal Candididiasis (VVC) in the Modern Era with Reducing Immunity by Different Candida Species Along with Increasing Resistance and Management -An Update

30. Zeng X., et al. "Risk factors of Vulvovaginal candidiasis among women of reproductive age in X'ian: a cross sectional study". BioMed Research International (2018): ID9703734.

31. Vermitsky JP., et al. "A survey of vaginal flora Candida species isolates from women of different age groups by use of species specific PCR detection". Journal of Clinical Microbiology 46.4 (2008):1501-1503.

32. Wadile R and Bhate V. "Study of clinical spectrum and risk factor of neonatal candidemia". Indian Journal of Pathology and Microbiology 46.4 (2008):1501-1503.

33. Ghaddar N., et al. "Emergence of Vulvovaginal candidiasis among Lebanese Pregnant women: Prevalence. risk factors, and species Distribution". Infectious Diseases in Obstetrics and Gynecology (2019): Art ID 5016810.

34. Amblee Y and Jeffery CJ. "Physical features of intracellular proteins thatmoonlight on the cell surface". PLOS ONE 10 (2015): e013o575.

35. Jeffery CJ. "Moonlighting proteins". Trends in Biochemical Sciences 24 (1999): 8-11.

36. Wang G., et al. "The roles of Moonlighting proteins in bacteria". Current Issues in Molecular Biology 16 (2014): 15-22.

37. Wisplinghoff H., et al. "Nosocomial bloodstream infections due to Candida spp.in the USA: species, duration, clinical features and antifungal susceptibilities". International Journal of Antimicrobial Agents 43 (2014): 78-81.

38. Kozik A., et al. "Fibronectin,vitronection and laminin binding proteins of the cell wall of Candida parapsilosis and Candida tropicalis pathogenic yeasts". BMC Microbiology 15 (2015): 197.

39. Karkowsa-Kuleta J., et al. "Binding of human plasminogen and high molecular weight kininogen by cell surface exposed proteins of candida parapsilosis". Acta Biochimica Polonica 64 (2017): 391-400.

40. Karkowsa-Kuleta J., et al. "Characterization of the intera ctions beweein human high molecular -mass kininogen and albicans cell wall protein s of pathogenic yeasts". Acta Biochimica Polonica 63 (2016): 427-36.

41. Lopez CM., et al. "Candida albicans uses the cell surface proteins Gpm1 to attach to human endothelial cells and to keratinocytes via the adhesive proteins vitronection tropicalis". PLOS ONE 9 (2014): e90796.
42. Nonez-Beltran A., et al. "Identification of proteins involved in the adhesion of Candida species to different medical devices". Microbial Pathogenesis 107 (2017): 293-303.

43. Serrano-F ujarte I., et al. "Moonlight-Like proteins of the cell wall protect protect cell wall sessile cells of Candida from oxidative stress". Microbial Pathogenesis 90 (2016): 22-33.

44. Hall RA. "Dressed toimpress: impact of environmental adaptation to the Candida albicans cell wall". Molecular Microbiology 97 (2015): 7-17.

45. Sadeghi G., et al. "Emergence of non Candida; albicans species: epidemiology, phylogeny and fluconazole susceptibility profile". Journal de Mycologie Médicale 28 (2018): 51-58.

46. Vialas V., et al. "Cell surface shaving of Candida albicans biofilms, hyphae and yeast form cells". Proteomics 12.14 (2012): 2331-2339.

47. Karkowsa-Kuleta J., et al. "Moonlighting proteins are variably exposed at the cell surfaces of Candida glabrata, Candida. parapsilosis and Candida tropicalis under certain growth conditions". BMC Microbiology 19 (2019): 149.

48. Larkin EL., et al. "A novel1,3 -Beta -d- glucan inhibitor, Ibrexafungerip 0 (Formerly SCY-078), shows Potent Activity in the Lower ph Environment of Vulvovaginitis". Antimicrobial Agents and Chemotherapy 63.5 (2019): e02611-e02618.

49. Abd Ellah NH., et al. "Efficacy of ketoconazole gel flakes in treatment of vaginal candidiasis: Formulation, in vitro and clinical evaluation". International Journal of Pharmaceutics 567 (2019): 118472.

50. Esposito E., et al. "Effect of pea protein plus grape seed dry extract on a murine model of Candida albicans induced vaginitis". Future Microbiology 13 (2018): 1375-1382.

\section{Volume 2 Issue 12 December 2019} (C) All rights are reserved by Kulvinder Kochar Kaur., et al. 